Discussion/conclusion The survey has demonstrated that the HIV testing policy has increased staff awareness and confidence in HIV testing. Late diagnosis rates continue to be monitored and misconceptions regarding HIV testing challenged.

\section{P239 DON'T ASK, DON'T TELL: INTRODUCTION OF ROUTINE DOMESTIC ABUSE PROMPT IN GENITOURINARY WALK- IN CLINIC}

Marie Williamson*, Frances Beanland, Andrea Mauger, Rachel Sacks, Alison Mears. Imperial College Healthcare NHS Trust, London, UK

\subsection{6/sextrans-2016-052718.285}

Background/introduction Domestic abuse (DA) is common and spontaneous disclosure rare. In July 2015, routine DA enquiry was introduced in a busy, inner London, walk-in genitourinary medicine clinic. All DA disclosures prompted completion of a proforma identifying ongoing risk/ $<3 / 12$ (=current DA) with clear management pathway. Patients disclosing current DA were seen by Sexual Health Information Protection (SHIP) team for detailed risk assessment and referrals, as needed. Those with previous DA were offered information/referrals if indicated.

Aim(s)/objectives To review DA disclosure management following DA routine prompt introduction.

Methods A retrospective case-notes review of patients' notes (coded DA) for 3 months from 14/07/2015.

Results 137 patients (111 women,26 men): age-range 17-75 years old(mean 33), 7\% (10/137) commercial sex workers, $11 \%$ (15/137) MSM/bisexual. $72 \%$ of patients had never disclosed to professionals previously. Ex-regular and current partners most common perpetrators (62\%, 15\% respectively). 25\% (34/137) disclosed current DA. Of these, 94\% (32/34) were offered SHIP referral, 88\% (28/32) accepted, 75\% (21/28) were seen. Referrals were made to DA services $48 \%(10 / 21)$, refuges $10 \%(2 / 21)$, MARAC 19\% (4/21). 52\% (11/21) had a standardised risk assessment; those who did not, 60\% (6/10) were already engaged in services, 20\% (2/10) didn't attend follow-up and $20 \%(2 / 10)$ referred to psychology. 44\% (14/34) patients were discussed at Vulnerable Adults MDT. No escalation of violence was recorded.

Discussion/conclusion Patients with significant risk from DA were identified who may not have disclosed without routine prompt. This is suggested as the majority were first disclosures. Interventions to reduce the negative consequences of DA for these patients have been performed.

\section{P240 WHAT IS THE HEPATITIS B VACCINATION COVERAGE IN MSM IN SOUTH WEST LONDON? AN AUDIT OF HEPATITIS B VACCINATION COVERAGE IN 'FIRST ATTENDEE' MSM IN A BUSY TEACHING HOSPITAL GUM CLINIC}

\footnotetext{
${ }^{1,2}$ Rachel Hill-Tout*, ${ }^{2}$ Holly Mitchell, ${ }^{2}$ Gwenda Hughes. ${ }^{1}$ St George's NHS Foundation Trust, London, UK; ${ }^{2}$ Public Health England, London, UK
}

\subsection{6/sextrans-2016-052718.286}

Background The 2001 National Strategy for Sexual Health and HIV recommended 90\% uptake of Hepatitis B (HBV) vaccine in non-immune MSM at first GUM clinic attendance. The HepB3 Survey reported 95\% uptake in 2008 but recent surveillance using GUM Clinic Activity Dataset-v2 (GUMCADv2) coding shows $<20 \%$ uptake. A detailed regional audit was designed to investigate this apparent drop in coverage.

Aim To determine HBV vaccination coverage in 'first-attendee' MSMs.

Methods All MSM 'first-attendees' at our service between January-March 2014 were identified. Patient records were reviewed for HBV screening, vaccine-offer, vaccine-uptake, HIV testing and coding accuracy up to 18 months from first-attendance. MSM were deemed 'immune' if surface antibody (sAb) $>10$ $\mathrm{mIU} / \mathrm{ml}$, core antibody positive, or self-reported vaccinationstatus was 'Fully-vaccinated' and no serology was done; and eligible for vaccination if $\mathrm{sAb} \leq 10 \mathrm{mIU} / \mathrm{ml}$, or if they reported 'Partially-vaccinated', 'Never-vaccinated', or 'Don't know' and no serology was done.

Results We identified 115 MSM 'first-attendees' (13 HIV+). 41\% only attended once. Regarding vaccination-status: 41/95 (43\%) reported 'Fully-vaccinated', $29 / 95$ (30\%) 'Partially-vaccinated', 12/95 (13\%)'Never-vaccinated', 11/95 (12\%) 'Don'tknow', 1/95 (1\%) 'Chronic-HBV' and 1/95 (1\%) 'Cleared-HBV'. 48/103 (47\%) were deemed immune and 46/103 (45\%) eligible. $36 / 46(76 \%)$ of eligibles were offered vaccination; 2/36 (6\%) declined, reporting 'not at risk'. $3 / 32$ (9\%) who accepted vaccination pending sAb levels did not return for it. 31/46 (67\%) of eligibles received $\geq 1$ dose of vaccine, 28/46 (61\%) within 42 days of first-attendance. Reasons for non-offer were not recorded. $75 \%$ of first-doses were coded. Only 15\% of 'immune' patients were coded as such (P2I). HIV-test uptake was $99 \%$ and coding accuracy was $97 \%$.

Discussion We found below-target levels of HBV vaccinationcoverage and incomplete coding of immunity/vaccination. Failure to code P2I for 'immunes' will increase the apparent 'eligibles' denominator in GUMCADv2 algorithms, generating incorrectly low vaccination-coverage figures. Reduced offer-rate may contribute to low vaccination-coverage and should be reviewed locally. Further regional audits may be required. Significant improvements in coding are essential for accurate surveillance of HBV vaccination-coverage using GUMCADv2.

\section{P241 DO MEN ATTENDING A GENITOURINARY MEDICINE SERVICE KNOW IF THEIR MOST RECENT SEXUAL PARTNER WAS USING CONTRACEPTION?}

William Gibson*, Amy Pearce, Frances Keane. Royal Cornwall Hospital, Truro, UK

\subsection{6/sextrans-2016-052718.287}

Background/introduction Unintended pregnancy is a significant problem. In 2014 in England and Wales 184,571 abortions were performed. Contraceptive methods generally focus on females. Males have the potential to contribute significantly to the contraceptive decisions in their relationships.

Aim(s)/objectives To demonstrate what knowledge male patients have of their partner's contraception status.

Methods Electronic proformas for male GUM patients in the region studied were amended to include a question that assessed whether or not the attending patient was aware if their most recent sexual partner (MRSP) was using contraception. After this was in place, notes of all male patients classified "new"/"rebook" who attended in January 2016 were studied. Those coded MSM (men who have sex with men) were excluded. An Excel workbook was created from the data of the 396 patients. Patients assessed on alternative proformas were excluded, as were those 